\title{
Standardization of Administered Activities in Pediatric Nuclear Medicine: A Report of the First Nuclear Medicine Global Initiative Project, Part 1-Statement of the Issue and a Review of Available Resources
}

\author{
Frederic H. Fahey ${ }^{1}$, Henry Hee-Seong Bom ${ }^{2}$, Arturo Chiti ${ }^{3}$, Yun Young $\mathrm{Choi}^{4}$, Gang Huang ${ }^{5}$, Michael Lassmann ${ }^{6}$, \\ Norman Laurin ${ }^{7}$, Fernando Mut ${ }^{8}$, Rodolfo Nuñez-Miller ${ }^{9}$, Darin O'Keeffe ${ }^{10}$, Prasanta Pradhan ${ }^{11}$, Andrew M. Scott ${ }^{12}$, \\ Shaoli Song ${ }^{13}$, Nischal Soni ${ }^{14}$, Mayuki Uchiyama ${ }^{15}$, and Luis Vargas ${ }^{16}$ \\ ${ }^{1}$ Boston Children's Hospital, Harvard Medical School, Boston, Massachusetts; ${ }^{2}$ Chonnam National University Hwasun Hospital, \\ Hwasun Jeonnam, South Korea; ${ }^{3}$ Humanitas Research Hospital, Rozzano, Italy; ${ }^{4}$ Hanyang University Hospital, Seoul, Republic of \\ Korea; ${ }^{5}$ Renji Hospital, School of Medicine, Shanghai Jiaotong University, Shanghai, China; ${ }^{6}$ University Würzburg, Würzburg, \\ Germany; ${ }^{7}$ Centre Hospitalier Affilié Universitaire Régional de Trois-Rivières, Quebec, Canada; ${ }^{8}$ Spanish Association Hospital, \\ Montevideo, Uruguay; ${ }^{9}$ International Atomic Energy Agency, Vienna, Austria; ${ }^{10}$ Christchurch Hospital, Christchurch, New Zealand; \\ ${ }^{11}$ Sanjay Gandhi Post Graduate Institute of Medical Sciences, Lucknow, India; ${ }^{12}$ Austin Hospital and Ludwig Institute for \\ Cancer Research, Melbourne, Australia; ${ }^{13}$ Renji Hospital, School of Medicine, Shanghai Jiaotong University, Shanghai, China; \\ ${ }^{14}$ Department of Nuclear Medicine, Inkosi Albert Luthuli Central Hospital, DurbanKwaZulu Natal, South Africa; ${ }^{15}$ Department of \\ Radiology, The Jikei University School of Medicine, Minato, Tokyo, Japan; and ${ }^{16}$ Xalapa Medical Center, Xalapa, Mexico
}

\begin{abstract}
The Nuclear Medicine Global Initiative (NMGI) was formed in 2012 and consists of 13 international organizations with direct involvement in nuclear medicine. The underlying objectives of the NMGl were to promote human health by advancing the field of nuclear medicine and molecular imaging, encourage global collaboration in education, and harmonize procedure guidelines and other policies that ultimately lead to improvements in quality and safety in the field throughout the world. For its first project, the NMGI decided to consider the issues involved in the standardization of administered activities in pediatric nuclear medicine. This article presents part 1 of the final report of this initial project of the NMGI. It provides a review of the value of pediatric nuclear medicine, the current understanding of the carcinogenic risk of radiation as it pertains to the administration of radiopharmaceuticals in children, and the application of dosimetric models in children. A listing of pertinent educational and reference resources available in print and online is also provided. The forthcoming part 2 report will discuss current standards for administered activities in children and adolescents that have been developed by various organizations and an evaluation of the current practice of pediatric nuclear medicine specifically with regard to administered activities as determined by an international survey of nuclear medicine clinics and centers. Lastly, the part 2 report will recommend a path forward toward global standardization of the administration of radiopharmaceuticals in children.
\end{abstract}

J Nucl Med 2015; 56:646-651

DOI: $10.2967 /$ jnumed.114.152249

Received Nov. 25, 2014; revision accepted Feb. 3, 2015.

For correspondence or reprints contact: Frederic H. Fahey, Division of Nuclear Medicine, Boston Children's Hospital, 300 Longwood Ave., Boston, MA 02115

E-mail: Frederic.Fahey@childrens.harvard.edu

Published online Mar. 12, 2015.

COPYRIGHT (c) 2015 by the Society of Nuclear Medicine and Molecular Imaging, Inc.
$\mathbf{I}$ summer of 2012, several international organizations directly involved in the practice and science of nuclear medicine decided to engage in a project of common interest for the betterment of the field worldwide. The underlying objectives were to promote human health by advancing the field of nuclear medicine and molecular imaging, encourage global collaboration in education, and harmonize procedure guidelines and other policies that ultimately lead to improvements in quality and safety in the field throughout the world. This endeavor was named the Nuclear Medicine Global Initiative (NMGI) and includes societies from various countries as well as several multinational organizations in the field of nuclear medicine (Table 1).

The 13 involved organizations determined that there would be great benefit to the field of nuclear medicine at large in developing a forum in which discussions of important topics of interest could be held. The group recognized that nuclear medicine is a relatively small medical specialty and that professional organizations in the specialty are challenged by limited time and resources. The NMGI is an opportunity for these professional organizations to leverage their strengths and expertise through international cooperation in areas of mutual interest and marks the first time international professional societies of nuclear medicine have worked together to achieve a specific goal.

It was decided that the group would consider several potential projects and select one that fulfilled certain criteria. It would need to be of common significant interest for all participants and achievable in a reasonable time frame. If such an endeavor were shown to be fruitful, then a second topic would be selected on completion of the first project.

Most of the NMGI participants met at the European Association of Nuclear Medicine meeting in Milan in October 2012. After a full discussion, the group decided that the first NMGI project would be to consider the issues involved in the standardization of administered activities in pediatric nuclear medicine. Several groups 
TABLE 1

NMGI Participants

\begin{tabular}{ll}
\hline \multicolumn{1}{c}{ Participating institution } & Representative \\
\hline Asia Oceania Federation of Nuclear Medicine and Biology & Henry Hee-Seong Bom \\
\hline Australian and New Zealand Society of Nuclear Medicine & Darin O'Keeffe and Andrew M. Scott \\
\hline Canadian Association of Nuclear Medicine & Norman Laurin \\
\hline Chinese Society of Nuclear Medicine & Gang Huang and Shaoli Song \\
\hline European Association of Nuclear Medicine & Arturo Chiti and Michael Lassmann \\
\hline International Atomic Energy Agency & Rodolfo Nuñez-Miller \\
\hline Japanese Society of Nuclear Medicine & Mayuki Uchiyama \\
\hline Korean Society of Nuclear Medicine & Yun Young Choi \\
\hline Latin American Association of Societies of Nuclear Medicine and Biology & Fernando Mut \\
\hline Society of Nuclear Medicine India & Prasanta Pradhan \\
\hline Society of Nuclear Medicine and Molecular Imaging & Frederic H. Fahey \\
\hline South African Society of Nuclear Medicine & Nischal Soni \\
\hline World Federation on Nuclear Medicine and Biology & Luis Vargas \\
\hline
\end{tabular}

have developed such guidelines that are clearly of value in the field; however, variations among these guidelines may cause confusion regarding their use, particularly for clinical sites that may not routinely perform imaging of children as well as for international groups seeking to establish their own guidelines. In addition, there is a large collection of educational and reference resources, both in print and online, that addresses the issues involved in the administration of radiopharmaceuticals to children that could be of significant value to nuclear medicine practitioners; thus, a catalog of such resources would be well regarded. Additionally, the group decided that a global snapshot of the current practice of pediatric nuclear medicine would be of considerable interest, and therefore an international survey was proposed. A working group was formed to develop and validate a survey instrument.

The report of the NMGI will be divided into 2 parts. This is the part 1 report, which provides a review of the value of pediatric nuclear medicine, the current understanding of the carcinogenic risk of radiation as it pertains to the administration of radiopharmaceuticals in children, and the application of dosimetric models in children. Gaps in current knowledge on these topics are also discussed. A listing of pertinent educational and reference resources available in print and online is provided. The part 2 report will discuss current standards for administered activities in children and adolescents that have been developed by various organizations and an evaluation of the current practice of pediatric nuclear medicine specifically with regard to administered activities as determined by an international survey of nuclear medicine clinics and centers. Lastly, the part 2 report will recommend a path forward toward global standardization of the administration of radiopharmaceuticals in children.

\section{LITERATURE REVIEW}

\section{Value of Pediatric Nuclear Medicine}

Nuclear medicine provides physiologic information with high sensitivity, and for this reason it is associated with a capability of detecting diseases at an early stage. Nuclear medicine imaging is commonly performed in adults and children, but the indications for pediatric nuclear medicine are often quite different from those for adults. Specifically, benign conditions tend to be more commonly examined than malignant diseases in children. In fact, approximately $90 \%$ of radionuclide studies in children are for nononcologic disorders, and almost one half of pediatric nuclear medicine tests are renal applications in most departments; this figure is similar across the world and has consistently been so over time (1).

Because of the higher radiosensitivity of biologic tissues in children as compared with adults, the radiation dose is a special challenge in this population, as it is for any other diagnostic procedure involving ionizing radiation. There is no standard recommended dose limit for patients; however, because the goal is to provide a better possible standard of care with the available resources in a specific environment, it is generally understood that the radiation dose to children should always be optimized for any necessary medical imaging. This is particularly important for young patients, not only because of radiosensitivity but also because the biologic effects of radiation usually appear long after the exposure, and thus the probability of adverse effects is proportional to the expected life span of the individual patient. Hence, all possible efforts should be made to limit the radiation dose to pediatric patients without compromising the diagnostic capabilities of the study $(1,2)$.

Pediatric nuclear medicine provides useful imaging modalities for pediatric patients in many acute care settings (3-6). Dorfman et al. reviewed more than 350,000 medical insurance records of children in the United States and determined that nuclear medicine comprised only $0.9 \%$ of radiologic examinations performed in children (4 nuclear medicine examinations per 1,000 children), the most common examinations being bone and thyroid scanning (7). Following are the descriptions of several applications commonly used in pediatric nuclear medicine. Renal imaging procedures are the most commonly performed in pediatric nuclear medicine. Hydronephrosis is a relatively common medical condition that ranges from a reversible dilation to obstructive nephropathy, and scintigraphic evaluation is a standard procedure in the management of this condition in children. The use of diuretics makes differentiation of functional and obstructive dilation easier. In addition, the use of captopril or another angiotensin-converting enzyme inhibitor is helpful in the detection of angiotensin up-regulated, which is a turning point for irreversible renal parenchymal injury and loss of renal function $(8,9)$. 
Renal cortical scintigraphy using ${ }^{99 \mathrm{~m}} \mathrm{Tc}$-dimercaptosuccinic acid (DMSA) has been a standard procedure for decades, particularly for the evaluation of pyelonephritis and detection of cortical scars after an acute episode. Interpretation of planar and SPECT images is well established and has low interobserver variation $(10,11)$.

Bone scintigraphy is the second most common procedure in radionuclide examination in children. It is a sensitive method to detect alteration of bone metabolism of any cause and should be correlated with the patient's clinical history and other test results because of its low specificity (12-14). Hybrid imaging with CT, such as SPECT/CT and PET/CT, improves specificity and diagnostic accuracy. It is helpful in localizing causes of pain in nonverbal children, small bone and soft-tissue lesions in trauma, infection, and tumors and therefore can be used to avoid hospital visits for patients and their parents. Additional radiation exposure from CT is an important consideration and can be avoided using individually tailored protocols and careful justification.

${ }^{123}$ I-metaiodobenzylguanidine (MIBG) scintigraphy for neuroblastoma has high specificity $(>90 \%)$ and is one of the most common methods of pediatric oncologic imaging. ${ }^{18} \mathrm{~F}-\mathrm{FDG} \mathrm{PET} / \mathrm{CT}$ is increasingly being used in neuroblastoma patients to overcome the limitations of poor spatial resolution and low sensitivity for ${ }^{123}$ I-MIBG (15), and almost every solid malignancy except brain tumors in children and adolescents has high avidity for ${ }^{18} \mathrm{~F}-\mathrm{FDG}$. Other PET tracers, including ${ }^{11} \mathrm{C}$-methionine, ${ }^{18} \mathrm{~F}-3$,4-dihydroxy6- ${ }^{18} \mathrm{~F}$-fluoro-L-phenylalanine, ${ }^{18} \mathrm{~F}-\mathrm{NaF},{ }^{68} \mathrm{Ga}-\mathrm{DOTATOC}$, and DOTANOC, are increasingly used in pediatric nuclear medicine but not as frequently as in adults (16).

Gastrointestinal bleeding from ectopic gastric mucosa in Meckel's diverticulum can be evaluated by ${ }^{99 \mathrm{~m}} \mathrm{Tc}$-pertechnetate scintigraphy (Meckel's scan) or ${ }^{99 \mathrm{~m}} \mathrm{Tc}$ red blood cell imaging. Hepatobiliary scintigraphy is the most accurate imaging test for acute cholecystitis and can also be used to evaluate biliary atresia in infants. A perfusion-ventilation lung scan is the first and safest imaging test for pediatric patients with suspected pulmonary embolism, with less radiation exposure than CT pulmonary angiography (5). Also, brain death can be diagnosed by brain scintigraphy. Finally, coronary arterial occlusion in patients with Kawasaki disease can be evaluated by myocardial perfusion imaging.

The identification of epileptogenic foci in children with refractory epilepsy represents a significant diagnostic challenge. MR imaging frequently fails to reveal an anatomic origin for the seizures, and scalp electroencephalography is often limited to identification of the involved hemisphere. Functional imaging modalities such as PET and SPECT are more useful tools for this application because they reflect the functional pathology associated with the seizure. Although these changes are more easily identified ictally, they can also be detected in the interictal phase, providing relevant information for surgical treatment $(17,18)$.

\section{Radiation Risk in Children}

Attempting to determine the risk of adverse health effects from exposure of ionizing radiation in the dose range commonly encountered in clinical nuclear medicine is a daunting task. It involves the application of models based on the most current knowledge of pertinent epidemiologic and biologic data. In practically all cases, the scientific data were acquired in a manner that does not exactly match that of interest in nuclear medicine, and thus it is necessary to extrapolate to the endeavor of interest (e.g., from high-dose data in humans or low-dose data in animals to low-dose effects in humans). Much of our basic understanding of the effects on human health resulting from exposure to ionizing radiation comes from the Life Span Study of the survivors of the bombings of Hiroshima and Nagasaki, as reported by the Radiation Effects Research Foundation (19-22). A recent evaluation of these data by Ozasa et al. indicated an increased risk of cancers of the stomach, lung, liver, colon, breast, gallbladder, esophagus, bladder, and ovary (23). However, no increased risk was found for cancers of the rectum, pancreas, uterus, prostate gland, and kidney. A total of 86,600 subjects were followed up for solid tumors from 1950 to 2003, and it was estimated that there were 527 excess deaths in that population. Several other epidemiologic studies have evaluated the risk of ionizing radiation in humans, and in general the results of these studies corroborate the findings of the Life Span Study.

A review of the data from the Life Span Study indicates a clear relationship between induction of solid cancer and radiation dose at levels greater than $0.5 \mathrm{~Gy}$. However, the limitations of epidemiologic approaches make it difficult to estimate the risk at the dose range associated with clinical nuclear medicine (i.e., 0.05-0.1 Gy). Differences in dose rate or the fractionation of dose between the epidemiologic subjects and nuclear medicine patients can also affect the accuracy of the estimation. For these reasons, the evaluation of findings from investigations in radiobiology can often provide valuable insight.

The National Academy of Sciences Committee on the Biologic Effects of Ionizing Radiation issued a report in 2007 that reviewed the state of knowledge in radiation epidemiology and biology at that time and developed models of radiation risk as a function of dose, sex, and age at the time of exposure. This review was published in what is referred to as the BEIR VII phase 2 report (24). This report recommended a linear no-threshold model for cancer induction by ionizing radiation in solid tumors and a linear quadratic model for leukemia. Although some controversies exist regarding the scientific validity of the linear no-threshold model for estimating radiation risk at low doses, it may be considered a conservative and thereby prudent model for radiation safety purposes. According to the models provided by the BEIR VII phase 2 report, those exposed at an earlier age are in general at higher risk for cancer induction from ionizing radiation than adults. For example, a 1-y-old child and a 10-y-old child may have an approximately 3 times and 2 times higher risk, respectively, of cancer induction than a 40-y-old adult for the same level of exposure. In addition, a young girl has a 30\%-40\% higher risk of cancer induction than a young boy with the same level of exposure, mostly due to the risk from breast cancer (Fig. 1) (25).

In 2013, the United Nations Scientific Committee on the Effects of Atomic Radiation (UNSCEAR) issued a report titled Sources, Effects and Risks of Ionizing Radiation (volume II, annex B) on the effects of radiation exposure in children (26). Their 2006 report to the General Assembly stated that lifetime cancer risk estimates for children are uncertain and may be 2-3 times higher than those for a population exposed at all ages based on a lifetime projection model that combined all tumor types, which was consistent with the risk estimate provided by the BEIR VII phase 2 report. However, the 2013 report stated that such generalizations may be misleading because the impression is that all types of tumors have similar radiosensitivity, which, as pointed out in the report, is not the case. The report included a review of data for 23 types of tumors in regard to the evidence as to whether there was an association with ionizing radiation and whether there was an effect based on the age of exposure (i.e., whether younger patients 


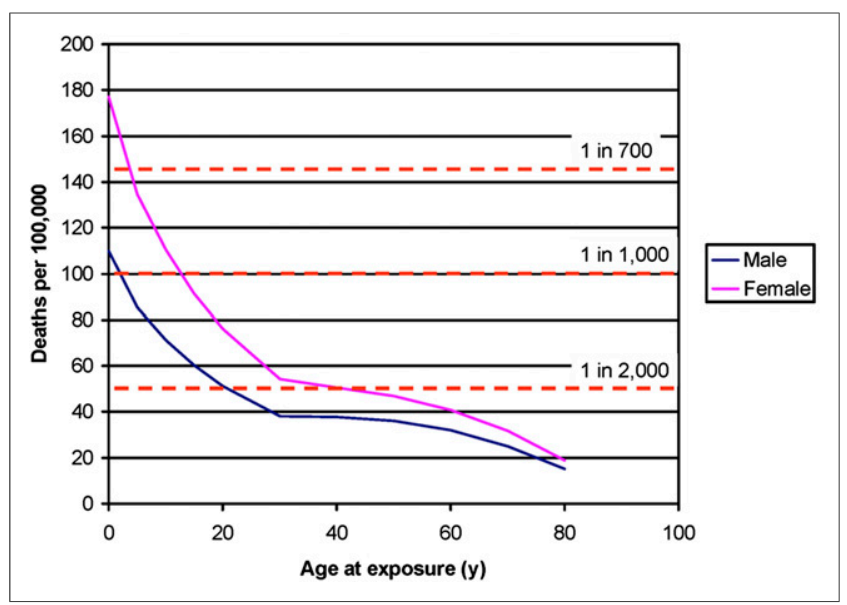

FIGURE 1. Lifetime attributable risk of cancer death as function of age at time of exposure and sex resulting from 10-mSv exposure based on BEIR VII phase 2 report (17). Dashed lines indicate attributable risk levels of 1 in 700,1 in 1,000 , and 1 in 2,000 corresponding to sex-averaged risk for 1-, $10-$, and 40-y-old, respectively. (Reprinted with permission of (25).)

were at higher or lower risk with the same level of exposure). The report indicated that $25 \%$ of the types of tumors (including leukemia as well as thyroid, skin, breast, and brain) clearly demonstrated higher radiation sensitivity in younger subjects. In $15 \%$ of the types of tumors (including bladder), children had the same level of radiosensitivity as adults. For another $10 \%$ of the types of tumors (most notably lung), the risk in younger subjects was lower than that in adults. In the other $50 \%$ of types of tumors, the association was either too weak to draw a conclusion regarding the relationship between risk and age (e.g., for the esophagus) or there was no evidence that a relationship between radiation and tumor induction at any age (Hodgkin lymphoma, prostate, rectum, or uterus) existed. For 2 types of tumors (leukemia and lung), associations of risk with age were notably different between the BEIR VII phase 2 report and the 2013 UNSCEAR report. There was little variation in risk at different ages in the BEIR VII phase 2 report, whereas there was a markedly higher risk for younger patients in the 2013 UNSCEAR report. Conversely, the BEIR VII phase 2 report indicated a higher risk, and the 2013 UNSCEAR report reported a slightly lower risk of lung cancer in children.

In summary, children may be considered in general to be at higher risk for adverse health effects from ionizing radiation than adults. Across many types of tumors, children may be 2-3 times more sensitive than adults. However, this is not true for all types of tumors; some may demonstrate higher radiosensitivity, some less radiosensitivity, and some radiosensitivity similar to that of adults. More data are necessary to provide reliable, tumor-specific risk estimates.

\section{Pediatric Dosimetric Models}

In the 2007 International Commission on Radiological Protection (ICRP) recommendations (ICRP 103), the basic definition of effective dose remained unchanged from the 1990 recommendations (ICRP 60) $(27,28)$. However, some of the tissue-weighting factors changed based on new epidemiologic data for cancer induction. In fact, instead of considering cancer mortality as a basis for tissue-weighting factors, the new recommendations use the incidence of radiation-induced cancer as well as the risk of heritable disease over the first 2 generations. The most significant changes in tissue-weighting factors are found for breast tissue, the gonads, and the remainder organs.
The ICRP mandates the use of male and female reference voxel phantoms, as published in ICRP 110 (29). This new concept demands a determination of equivalent doses in the organs and tissues of the reference male and the reference female separately. To obtain the equivalent doses of the reference person, the sexspecific equivalent doses are averaged; hence, the new tissueweighting factors can be applied. Moreover, according to ICRP 103, only the latest ICRP voxel phantoms have to be used for calculations of effective dose. Applying the new weighting factors on a set of equivalent organ doses previously calculated with a mathematic phantom will therefore not result in a correct effective dose value due to ICRP 103. Presently, the modified tissue-weighting factors and the subsequent calculation of the effective dose according to the formalism of ICRP 103 cannot directly be applied to nuclear medicine because the calculations of the $S$ values for the radiopharmaceuticals using the new recommendations of the ICRP are yet to be developed but should be available soon.

\section{Dosimetry for Commonly Performed Procedures in Children}

In 2011, as part of the PEDDOSE.NET research project of the European Union (www.peddose.net), an overview of data on dosimetry for the most commonly used diagnostic radiopharmaceuticals was published as a basis for subsequent recommendations $(30,31)$. In summary, data on dosimetry are available for many commonly used radiopharmaceuticals, although the data collection and calculation methods were heterogeneous, and most of the data were acquired more than 20 y ago. However, data on biokinetics or absorbed doses for pediatric nuclear medicine applications calculated with clinical data are missing in most cases (31).

Much of the data on dosimetry for children and adolescents published by the ICRP uses information on biokinetics from adults (32-34). The organ residence time (i.e., the product of the fraction of the radiopharmaceutical reaching the organ and the effective mean time is in the organ) for adults was used as an input for calculating absorbed doses for children and adolescents using agedependent (i.e., size- and weight-dependent) mathematic phantoms. Thus, a potential age dependency in pharmacokinetics was not considered in much of the data provided by the ICRP. For example, the data on ${ }^{99 \mathrm{~m} T c-D M S A}$ were collected more than $30 \mathrm{y}$ ago and have not been updated. In particular, the age-dependent data on effective dose values in ICRP publication 80 (33) rely on those datasets. Therefore, there might be a larger error associated with the effective dose as compared with other radiopharmaceuticals.

In 2011, Sgouros et al. reported on an approach for balancing diagnostic image quality with cancer risk for ${ }^{99 m}$ Tc-DMSA (35). The authors used pharmacokinetic modeling and a pediatric series of nonuniform phantoms for simulating ${ }^{99 \mathrm{~m} T c-D M S A ~ S P E C T}$ images. Images were generated for several different administered activities and for several lesions with different target-to-background activity concentration ratios; the phantoms were also used to calculate organ $\mathrm{S}$ values for ${ }^{99 \mathrm{~m}} \mathrm{Tc}$. An analysis of the diagnostic quality of images was performed with different modeled administered activities (i.e., count densities) for anthropomorphic reference phantoms representing two 10 -y-old girls with equal weights but different body morphometry. Using BEIR VII ageand sex-specific risk factors, the authors converted absorbed doses to excess risk of cancer incidence and used the values to directly assess the risk of the procedure. This study illustrates that the implementation of a more rigorous approach for balancing the benefits of adequate image quality against the risks of radiation may be preferable to the use of effective dose and also demonstrates 
TABLE 2

Strengths and Limitations of Current Educational Content

\begin{tabular}{ll}
\hline \multicolumn{1}{c}{ Strengths } & \multicolumn{1}{c}{ Limitations } \\
\hline Most content is free (open access) on the Internet & Language barrier (available in English only) \\
\hline Wide range of topics covered & Some important topics do not have adequate coverage \\
Fairly up to date & Heterogeneous platforms or displays \\
$\begin{array}{l}\text { Trustworthy sources with minimal or limited commercial } \\
\text { interest }\end{array}$ & $\begin{array}{c}\text { Lack of global guidelines. Recent European Association of Nuclear } \\
\text { Medicine (EANM)/Society of Nuclear Medicine and Molecular } \\
\text { Imaging (SNMMI) harmonization is a step in the right direction }\end{array}$ \\
\hline
\end{tabular}

that weight-based adjustment to the administered activity might be suboptimal.

\section{Use of Concept of Effective Dose in Children}

According to ICRP 103, effective dose is a protection quantity that provides a dose value related to the probability of health detriment to an adult reference person due to stochastic effects from exposure to low doses of ionizing radiation $(27,28,36)$. Although references such as ICRP $106(34)$ provide conversion factors ( $\mathrm{mSv}$ per $\mathrm{MBq}$ ) for pediatric models, it is a problematic quantity for use in children whose radiosensitivity may be higher than that for adults as previously described. In particular, the effective dose reflects the risk of the nonuniform dose distribution in terms of a uniform or whole-body exposure. This is important for medical applications, because most medical exposures consist of nonuniform partial body irradiations.

For comparison of different diagnostic procedures or similar procedures in different hospitals and countries, the effective dose can be useful. Furthermore, it is a good quantity to compare the use of different technologies for the same medical examination. However, this is true only for patient populations with the same age and sex distribution (27). For this reason, the effective dose should not be used for epidemiologic studies or an individual dose and risk assessment $(27,37)$.

\section{EDUCATIONAL RESOURCES IN PEDIATRIC DOSE OPTIMIZATION}

Because of recent public awareness and concerns regarding increasing radiation exposure from medical imaging examinations, this issue has received special attention from several medical professional organizations, industry, patient advocacy groups, and even the media. Although there remains some level of uncertainty regarding dosimetric models and the magnitude of the risk to radiation, making it prudent to adopt a cautious approach and to further our scientific knowledge on the subject, it is equally important to translate the current scientific findings and understanding into educational and informational resources for use in the clinic. As a result, there are a rising number of educational materials available on the specialized topic of dose optimization in pediatric nuclear medicine. A catalog of these resources is provided as online reference material for this article (Supplemental Table 1; supplemental materials are available at http://jnm.snmjournals.org). These include further refinement of existing guidelines, harmonization between existing guidelines, new investigations, and development of several new Internet-based web portals containing information and educational materials about dose optimization in pediatric nuclear medicine. Fortunately, most of this educational material can be accessed by both professionals and the general public.
When reviewing these educational materials, it is sometimes difficult to determine the intended target audience for a particular publication or web page (Table 2). Most of the information is accessible via the Internet and is in open-access format. The type of content is fairly varied, ranging from classic papers to guidelines, press releases from professional societies, webinars, radiopharmaceutical dose calculation web pages, newsletters, and online slide presentations. There is not a uniform standard of presentation style or format, and for some of the online lectures there is a fee to obtain access. Some of the web portals, such as the one for Image Gently, are more elaborate, providing information/ educational resources for parents, radiologists, technologists, and referring physicians (imagegently.com). Most, if not all, of this educational material is available in English only. Although the available educational content is considerably extensive and continues to grow, there are gaps in knowledge and educational content, such as optimization protocols for the CT component of hybrid imaging modalities or further development in regard to dose optimization through the establishment of clinical guidelines for pediatric imaging with SPECT/CT.

For future development, it is important to have the educational content available in several of the most frequently spoken languages in the world. In addition, streamlining the presentation style and web platform used for the educational content would be helpful.

Encouraging the inclusion of radiation dose optimization of medical imaging in general and nuclear medicine in particular in medical school and residency training curricula will help bring needed attention to this important topic. As new technologic developments are introduced in medical imaging, the current guidelines for dose optimization in pediatric nuclear medicine will most likely need to be revised and updated. Therefore, the presentation of educational content will need to be flexible to allow updates and changes in the future.

\section{CONCLUSION}

In this part 1 report, a literature review was performed of application, dosimetry, and radiation risk associated with pediatric nuclear medicine. In addition, a review of the educational and informational resources available in print or online is presented. This report sets the stage for the part 2 report, which will present a review and comparison of existing guidelines for administered activities in children and adolescents and the results of a global survey on the current state of the practice of pediatric nuclear medicine. The part 2 report will also present conclusion and recommendations of the initial project of the NMGI.

The application of nuclear medicine in the pediatric population has been shown to be of great value in a variety of clinical 
applications including cardiology, urology, neurology, orthopedics, and oncology. However, it is well believed that children are at a higher risk for adverse effects from ionizing radiation than adults, and, thereby, it is prudent to be more cautious when determining the administered activities for studies in children. This can be challenging because the dosimetric models available for children and adolescents are not as well established as they are in adults, particularly with respect to biodistribution of radiopharmaceuticals. There are a variety of educational and informational resources regarding the practice, dosimetry, and radiation risk associated with pediatric nuclear medicine. This report provides a list of links to many such resources that should be of clear value to the nuclear medicine practitioner.

\section{ACKNOWLEDGMENTS}

We thank the leadership and staff of the participating organizations listed in Table 1 without whose enthusiastic support this project would not have been possible. We also thank Andrea Bauer and Henrik Silber of the EANM for assisting with the faceto-face meetings held in conjunction with the EANM Annual Meeting. We specifically thank Virginia Pappas, Nikki WenzelLamb, and Rachel Woodson of the SNMMI for providing the infrastructure for the NMGI, which is not simple with 13 organizations across the globe. Conference calls were particularly challenging. Last, we thank Rebecca Maxey and Karen Deitz of the SNMMI for their editorial help with this manuscript.

\section{REFERENCES}

1. Conway JJ. Quo vadis pediatric nuclear medicine. Semin Nucl Med. 2007; 37:242-248.

2. Treves ST, Falone AE, Fahey FH. Pediatric nuclear medicine and radiation dose. Semin Nucl Med. 2014;44:202-209.

3. Treves ST, ed. Pediatric Nuclear Medicine and Molecular Imaging. 4th ed. New York, NY: Springer; 2014.

4. Charon M. Pediatric PET Imaging. New York, NY: Springer; 2006

5. Shammas A, Vali R, Charron M. Pediatric nuclear medicine in acute care. Semin Nucl Med. 2013;43:139-156.

6. Nadel HR. SPECT/CT in pediatric patient management. Eur J Nucl Med Mol Imaging. 2014;41:S104-S114.

7. Dorfman AL, Fazel R, Einstein AJ, et al. Use of medical imaging procedures with ionizing radiation in children. Arch Pediatr Adolesc Med. 2011;165:458-464.

8. Zucchetta P, Artifoni L, Rigamonti W, Cecchin D, Bui F, Murer L. Molecular biology and nuclear medicine in pediatric hydronephrosis. $Q \mathrm{~J} \mathrm{Nucl} \mathrm{Med} \mathrm{Mol}$ Imaging. 2010;54:363-371.

9. Piepsz A, Ham HR. Pediatric applications of renal nuclear medicine. Semin Nucl Med. 2006;36:16-35.

10. Craig JC, Irwig L, Ford M, et al. Reliability of DMSA for the diagnosis of renal parenchymal abnormality in children. Eur J Nucl Med. 2000;27:1610-1616.

11. Edefonti A, Tel F, Testa S, De Palma D. Febrile urinary tract infections: clinical and laboratory diagnosis, imaging and prognosis. Semin Nucl Med. 2014;44: 123-128.

12. Nadel HR. Pediatric bone scintigraphy update. Semin Nucl Med. 2010;40:31-40.

13. Ma JJ, Kang BK, Treves ST. Pediatric musculoskeletal nuclear medicine. Semin Musculoskelet Radiol. 2007;11:322-334.

14. Shammas A. Nuclear medicine imaging of the pediatric musculoskeletal system. Semin Musculoskelet Radiol. 2009;13:159-180.
15. Mueller WP, Coppenrath E, Pfluger T. Nuclear medicine and multimodality imaging of pediatric neuroblastoma. Pediatr Radiol. 2013;43:418-427.

16. Kumar R, Shandal V, Shamim SA, Halanaik D, Malhotra A. Clinical applications of PET and PET/CT in pediatric malignancies. Expert Rev Anticancer Ther. 2010;10:755-768.

17. Packard AB, Roach PJ, Davis RT, et al. Ictal and interictal technetium-99mbicisate brain SPECT in children with refractory epilepsy. $\mathrm{J}$ Nucl Med. 1996;37:1101-1106.

18. Kumar A, Chugani HT. The role of radionuclide imaging in epilepsy, part 1: sporadic temporal and extratemporal lobe epilepsy. J Nucl Med. 2013;54:17751781 .

19. Preston DL, Shimizu Y, Pierce DA, et al. Studies of mortality of atomic bomb survivors: report 13-solid cancer and noncancer disease mortality: 1950-1997. Radiat Res. 2003;160:381-407.

20. Preston DL, Pierce DA, Shimizu Y, et al. Effect of recent changes in atomic bomb survivor dosimetry on cancer mortality risk estimates. Radiat Res. 2004;162:377-389.

21. Preston DL, Cullings H, Suyama A, et al. Solid cancer incidence in atomic bomb survivors exposed in utero or as young children. J Natl Cancer Inst. 2008;100: 428-436.

22. Little MP. Heterogeneity of variation of relative risk by age at exposure in the Japanese atomic bomb survivors. Radiat Environ Biophys. 2009;48:253-262.

23. Ozasa K, Shimizu Y, Suyama A, et al. Studies of the mortality of atomic bomb survivors, Report 14, 1950-2003: an overview of cancer and noncancer diseases. Radiat Res. 2012;177:229-243.

24. Committee to Assess. Health Risks from Exposure to Low Levels of Ionizing Radiation: BEIR VII Phase 2. Washington, DC: National Research Council of the National Academies; 2006.

25. Fahey FH, Treves ST, Adelstein SJ. Minimizing and communicating radiation risk in pediatric nuclear medicine. J Nucl Med. 2011;52:1240-1251.

26. United Nations Scientific Committee on the Effects of Atomic Radiation (UNSCEAR). UNSCEAR 2013 Report Vol. II. Sources, Effects and Risks of Ionizing Radiation. Annex B. New York, NY: United Nations; 2013.

27. International Commission on Radiological Protection (ICRP). The 2007 recommendations of the International Commission on Radiological Protection. ICRP publication 103. Ann ICRP. 2007;37:1-332.

28. International Commission on Radiological Protection (ICRP). 1990 recommendations of the International Commission on Radiological Protection. ICRP publication 60. Ann ICRP. 1991;21:1-201.

29. Menzel HG, Clement C, DeLuca P. Realistic reference phantoms: an ICRP/ICRU joint effort-a report of adult reference computational phantoms. Ann ICRP. 2009;39:1-164.

30. Eberlein U, Broer JH, Vandevoorde C, et al. PEDDOSE.NET: dosimetry and health effects of diagnostic applications of radiopharmaceuticals with particular emphasis on the use in children and adolescents [abstract]. Eur J Nucl Med Mol Imaging. 2011;38:S438.

31. Eberlein U, Broer JH, Vandevoorde C, et al. Biokinetics and dosimetry of commonly used radiopharmaceuticals in diagnostic nuclear medicine: a review. Eur J Nucl Med Mol Imaging. 2011;38:2269-2281.

32. International Commission on Radiological Protection (ICRP). Radiation dose to patients from radiopharmaceuticals. ICRP publication 53. Ann ICRP. 1988;18.

33. International Commission on Radiological Protection (ICRP). Radiation dose to patients from radiopharmaceuticals (addendum 2 to ICRP publication 53). ICRP publication 80. Ann ICRP. 1998;28:1-126.

34. International Commission on Radiological Protection (ICRP). Radiation dose to patients from radiopharmaceuticals: addendum 3 to ICRP publication 53. ICRP. publication 106. Ann ICRP. 2008;38:1-197.

35. Sgouros G, Frey EC, Bolch WE, Wayson MB, Abadia AF, Treves ST. An approach for balancing diagnostic image quality with cancer risk: application to pediatric diagnostic imaging of ${ }^{99 \mathrm{~m}} \mathrm{Tc}$-dimercaptosuccinic acid. J Nucl Med. 2011;52:1923-1929.

36. Martin CJ. Effective dose: how should it be applied to medical exposures? $\mathrm{Br} \mathrm{J}$ Radiol. 2007;80:639-647.

37. Hendrick RE. Radiation doses and cancer risks from breast imaging studies. Radiology. 2010;257:246-253. 